\title{
The physiological determinants of near- infrared spectroscopy-derived regional cerebral oxygenation in critically ill adults
}

\author{
Michael D. Wood', Jill A. Jacobson², David M. Maslove ${ }^{3,4}$, John G. Muscedere', J. Gordon Boyd ${ }^{1,3,4^{*}}$ and The
} Cerebral Oxygenation and Neurological Outcomes Following Critical Illness (CONFOCAL) Research Group

\author{
* Correspondence: \\ gordon.boyd@kingstonhsc.ca \\ ${ }^{1}$ Centre for Neuroscience Studies, \\ Queen's University, 18 Stuart St, \\ Botterell Hall, Kingston, ON, Canada \\ ${ }^{3}$ Department of Critical Care \\ Medicine, Queen's University, Rm \\ 22.2.359 Davies 2, Kingston General \\ Hospital, 76 Stuart St, Kingston, ON \\ K7L 2V7, Canada \\ Full list of author information is \\ available at the end of the article
}

\begin{abstract}
Background: To maintain adequate oxygen delivery to tissue, resuscitation of critically ill patients is guided by assessing surrogate markers of perfusion. As there is no direct indicator of cerebral perfusion used in routine critical care, identifying an accurate strategy to monitor brain perfusion is paramount. Near-infrared spectroscopy (NIRS) is a non-invasive technique to quantify regional cerebral oxygenation $\left(\mathrm{rSO}_{2}\right)$ that has been used for decades during cardiac surgery which has led to targeted algorithms to optimize $\mathrm{rSO}_{2}$ being developed. However, these targeted algorithms do not exist during critical care, as the physiological determinants of $\mathrm{rSO}_{2}$ during critical illness remain poorly understood.
\end{abstract}

Materials and methods: This prospective observational study was an exploratory analysis of a nested cohort of patients within the CONFOCAL study (NCT02344043) who received high-fidelity vital sign monitoring. Adult patients ( $\geq 18$ years) admitted $<24 \mathrm{~h}$ to a medical/surgical intensive care unit were eligible if they had shock and/or required mechanical ventilation. Patients underwent $\mathrm{rSO}_{2}$ monitoring with the FORESIGHT oximeter for $24 \mathrm{~h}$, vital signs were concurrently recorded, and clinically ordered arterial blood gas samples and hemoglobin concentration were also documented. Simultaneous multiple linear regression was performed using all available predictors, followed by model selection using the corrected Akaike information criterion (AICC).

Results: Our simultaneous multivariate model included age, heart rate, arterial oxygen saturation, mean arterial pressure, $\mathrm{pH}$, partial pressure of oxygen, partial pressure of carbon dioxide $\left(\mathrm{PaCO}_{2}\right)$, and hemoglobin concentration. This model accounted for a significant proportion of variance in $\mathrm{rSO}_{2}\left(R^{2}=0.58, p<0.01\right)$ and was significantly associated with $\mathrm{PaCO}_{2}(p<0.05)$ and hemoglobin concentration $(p<0.01)$. Our selected regression model using AICc accounted for a significant proportion of variance in $\mathrm{rSO}_{2}\left(R^{2}=0.54, p<0.01\right)$ and was significantly related to age $(p<0.05), \mathrm{PaCO}_{2}(p<0.01)$, hemoglobin $(p<0.01)$, and heart rate $(p<0.05)$.

Conclusions: Known and established physiological determinants of oxygen delivery accounted for a significant proportion of the $\mathrm{rSO}_{2}$ signal, which provides evidence that NIRS is a viable modality to assess cerebral oxygenation in critically ill adults. Further elucidation of the determinants of $\mathrm{rSO}_{2}$ has the potential to develop a NIRS-guided resuscitation algorithm during critical illness.

(Continued on next page) 
(Continued from previous page)

Trial registration: This trial is registered on clinicaltrials.gov (Identifier: NCT02344043), retrospectively registered January 8, 2015.

Keywords: Near-infrared spectroscopy, Critical illness, Brain tissue oxygenation, Cerebral oximetry, Cerebral perfusion

\section{Background}

The resuscitation phase (i.e., first 24-48 h) of critical illness is directed at maintaining adequate oxygen delivery to tissues to end-organ injury. Several organ systems have objective surrogate markers that can be serially monitored to ensure optimal end-organ perfusion. For example, renal perfusion is monitored by serially measuring urine output and serum creatinine levels [1]. In contrast, there is currently no well-defined proxy of cerebral perfusion used in routine clinical practice. This constitutes an important gap in our understanding of critical illness, as neuronal ischemia is a universal pathological finding in patients who die in the intensive care unit (ICU) [2]. In the absence of objective, quantitative markers, clinicians in the ICU rely upon the neurological exam (e.g., evaluating alertness, orientation, and ability to follow motor commands) [3] to assess brain perfusion. This clinical exam is often confounded by sedation, analgesia, and severity of illness. Therefore, clinical assessments may be unreliable in this setting and an alternative strategy to accurately monitor cerebral perfusion is needed to prevent irreversible neuronal injury.

Near-infrared spectroscopy (NIRS) is a simple and non-invasive technique to quantify regional cerebral oxygenation $\left(\mathrm{rSO}_{2}\right)$. An adhesive sensor and light source are placed on the forehead, which emit varying wavelengths of infrared light (e.g., 700-1000 nm) that pass through the skin and bone with minimal absorption to an approximate depth of $2-3 \mathrm{~cm}$ of cerebral tissue [4]. The light that returns to the sensor represents the amount of spectral absorption occurring in the tissue bed (i.e., changes in oxygenatedand deoxygenated-hemoglobin), with venous circulation accounting for the majority of the signal (75-80\%) [5], and is typically displayed as an absolute value ranging from 0 to $99 \%$. The NIRS signal correlates with other measures of brain perfusion (e.g., jugular venous bulb oxygen saturation [6], brain tissue oxygen tension [7], and CT perfusion [8]), and its feasibility in critical care research has already been demonstrated [9].

Furthermore, NIRS has been described for many years in the cardiac anesthesiology literature. A targeted algorithm to optimize $\mathrm{rSO}_{2}$ during cardiac surgery has been developed $[10,11]$, which offers the potential to detect changes in cerebral perfusion and guide clinician intervention, and it has been demonstrated that nearly every episode (i.e., 97\%) of cerebral desaturation can be successfully reversed [12]. However, outside of the operating room, the physiological determinants of cerebral oxygenation are poorly understood. As a result, targeted algorithms do not currently exist to optimize $\mathrm{rSO}_{2}$ in the ICU. This may be relevant, as we have recently demonstrated that low $\mathrm{rSO}_{2}$ is an independent risk factor for the subsequent development of delirium in critically ill patients [13]. A nested cohort within that prospective observational study underwent high-fidelity vital sign monitoring. The objective of the present study was to define the hemodynamic and physiological determinants of the NIRS-derived $\mathrm{rSO}_{2}$ from that nested cohort of critically ill patients. 


\section{Material and methods}

\section{Study design and recruitment}

The Cerebral Oxygenation and Neurological outcomes FOllowing CriticAL illness (CONFOCAL) study (NCT02344043 clinicaltrials.gov) was a single-center prospective observational study $(n=103)$ for which the full protocol has been previously published [14]. As our protocol advanced from feasibility to a single-center pilot study, so did our data collection strategy, which initially began as hourly recordings documented in the electronic health records and advanced to continuous vital sign monitoring described below. The current manuscript is an exploratory analysis of a nested cohort of patients $(n=43)$ who received high-fidelity vital signs monitoring throughout the CONFOCAL study. Briefly, adult patients ( $\geq 18$ years) admitted $<24 \mathrm{~h}$ to a 33-bed general medical/ surgical and trauma ICU were eligible if they required mechanical ventilation with an expected duration $>24 \mathrm{~h}$ and/or having shock of any etiology. Shock was defined by vasopressor requirement at pre-specified doses [14]. Participants were excluded if they had a life expectancy $<24 \mathrm{~h}$, a pre-ICU diagnosis of cognitive dysfunction as indicated by their medical records, or a primary central nervous system diagnosis (e.g., traumatic brain injury).

\section{Data acquisition: $\mathrm{rSO}_{2}$, vital sign monitoring, and blood gas collection}

Immediately following enrolment, patients underwent $\mathrm{rSO}_{2}$ monitoring with the FORESIGHT monitor (CASMED, Caster Medical, Canada). For the majority of patients, a single $5-\mathrm{cm}$ sensor was placed on the center of the patients' forehead, $>3 \mathrm{~cm}$ from the superior rim of the orbit to avoid the frontal sinus [15], and recorded for $24 \mathrm{~h}$. As the more traditional sensor placement used for patients undergoing cardiac surgery is bilaterally on the frontal lobes [16], a subset of CONFOCAL patients $(n=10)$ received an additional bilateral sensor (right) to quantify the level of agreement between the two NIRS sensors. This analysis indicated that the sensors shared an acceptable level of agreement (see Additional file 1: Figure S1) and that one sensor was adequate. These $\mathrm{rSO}_{2}$ recordings were not revealed to the treating clinicians. To assess the relationships among patient hemodynamics with the $\mathrm{rSO}_{2}$ recordings, we used commercially available software (Bedmaster, Excel Medical Electronics, FL, USA) to simultaneously capture the following high-frequency vital signs: heart rate (HR), arterial oxygen saturation $\left(\mathrm{SpO}_{2}\right)$, systolic and diastolic blood pressure, and mean arterial pressure (MAP). These data were captured locally and stored on dedicated servers at the Queen's University Centre for Advanced Computing (www.cac.queensu.ca). We also documented arterial and central venous blood gases, as well as hemoglobin concentration $(\mathrm{Hb})$, when ordered clinically throughout this $24-\mathrm{h}$ period of recording. As the first $24-48 \mathrm{~h}$ of critical care are guided at resuscitation of the patient, we chose to record for the first $24 \mathrm{~h}$ a patient's ICU stay as understanding the determinants of $\mathrm{rSO}_{2}$ during this crucial period are foundational to the future development of targeted algorithms to optimize cerebral oxygenation.

Data cleaning: detecting and editing data abnormalities

As databases containing high-frequency vital sign recordings are known to contain artifacts [17], we undertook data validation and cleaning steps to minimize the inclusion of 
these in our analysis. We removed missing data, as well as outliers based on cutoffs determined by inspection of histograms, see Additional file 2: Figure S2. Specifically, we removed $\mathrm{HR}$ values $<44$ or $>134$, MAP $>130$ or $<39, \mathrm{SpO}_{2}<80$, and $\mathrm{rSO}_{2}<50$ or $>$ 85 . We also removed data that were logically inconsistent, such as measures where the diastolic pressure was higher than the systolic or when values equaled 0 .

\section{Data analysis}

\section{Determinants of the NIRS-derived $\mathrm{rSO}_{2}$ signal}

All statistical analyses were performed using the $\mathrm{R}$ software version 3.3.2 [18]. Due to the variability of data collection (i.e., high frequency vs clinically ordered) presented above, all physiological variables were condensed to a 24 -h mean prior to regression analysis in order to ensure that the data included were independent values rather than repeated recordings, which would violate the independence assumption of linear regression. Our primary objective was to assess the hemodynamic and physiological variables (i.e., determinants of oxygen delivery) predicting $\mathrm{rSO}_{2}$ during the first $24 \mathrm{~h}$ of critical illness. Therefore, simultaneous multiple linear regression was performed using $\mathrm{HR}, \mathrm{SpO}_{2}, \mathrm{MAP}$, arterial $\mathrm{pH}$, arterial partial pressure of oxygen $\left(\mathrm{PaO}_{2}\right)$, arterial partial pressure of carbon dioxide $\left(\mathrm{PaCO}_{2}\right)$, and $\mathrm{Hb}$ as predictors. Although systolic and diastolic blood pressures were recorded, we only included MAP due to redundancy. As increasing age has been associated with decreases in cerebral blood flow (CBF) [19], and potentially $\mathrm{rSO}_{2}$, we included age as a covariate. A correlation matrix illustrating the various relationships between the predictors of $\mathrm{rSO}_{2}$ can be observed in Additional file 3: Figure S3. Due to potential overfitting, model selection was implemented to generate a reduced multivariate regression model using the MuMIn package for $\mathrm{R}$ [20]. This package was used to iteratively compare all possible models given the data, whereas other model selection techniques may drop predictors in a stepwise fashion (i.e., backwards or forwards) [21] and only evaluate a small fraction of all possible subsets of the data. Due to our relatively small sample size potentially biasing our analysis, we applied the corrected AIC method $\left(\mathrm{AIC}_{\mathrm{c}}\right)$ [22] instead of implementing the Akaike's information criterion (AIC) [23] as the model criterion. The lowest $\mathrm{AIC}_{\mathrm{c}}$ value represents the most parsimonious model accounting for a large amount of variance with as few predictors as possible, so as not to over- or under-fit the model while minimizing information loss. Diagnostic testing indicated that the residuals were normally distributed, had equal variances, and did not suggest substantial evidence of collinearity among predictors (data not shown). ${ }^{1}$ The regression model and individual predictors were considered statistically significant if $p<0.05$. The simultaneous and the selected regression model were compared using AIC.

\section{Sensitivity power analysis}

We performed a sensitivity power analysis to determine the minimum effect size that our regression analysis, based on model selection using AICc, could have detected given the data. We used the following model parameters: sample size $=43$, power $=$ 0.90 , number of predictors $=4$, and $\alpha=0.05$, which indicated that we could have detected a minimum effect of $R^{2}=0.289$. The sensitivity power analysis was performed using G*Power [24] version 3.1.9.2. 


\section{Results}

\section{Patient characteristics}

From March 2014 to September 2016, 1155 patients were assessed for eligibility and 104 were enrolled. Of this cohort $(n=103), 56$ patients (54\%) underwent high-frequency vital sign monitoring from August 2015 to September 2016, 7 of which $(13 \%)$ were excluded because they did not have an arterial line present. Of the remaining 49 patients, 6 were excluded due to missing or insufficient arterial blood gas data, resulting in the inclusion of 43 subjects in the regression analysis (Fig. 1). The primary admitting diagnoses were mainly respiratory failure (30\%), followed by severe sepsis/septic shock (16\%), cardiac (16\%), and gastrointestinal (16\%), whereas previous comorbidities largely included a history of hypertension (56\%), cardiac complications (44\%), and respiratory disease (33\%). Most patients were intubated at the time of enrollment (95\%), and approximately half (56\%) were being treated with vasoactive agents. The median age was 68 years (IQR, 58.5-79), most patients were male (67\%), median length of ICU stay was 7 days (IQR, 4 to 13), and ICU mortality was 7 (16\%). Full demographics and clinical characteristics are shown in Table 1.

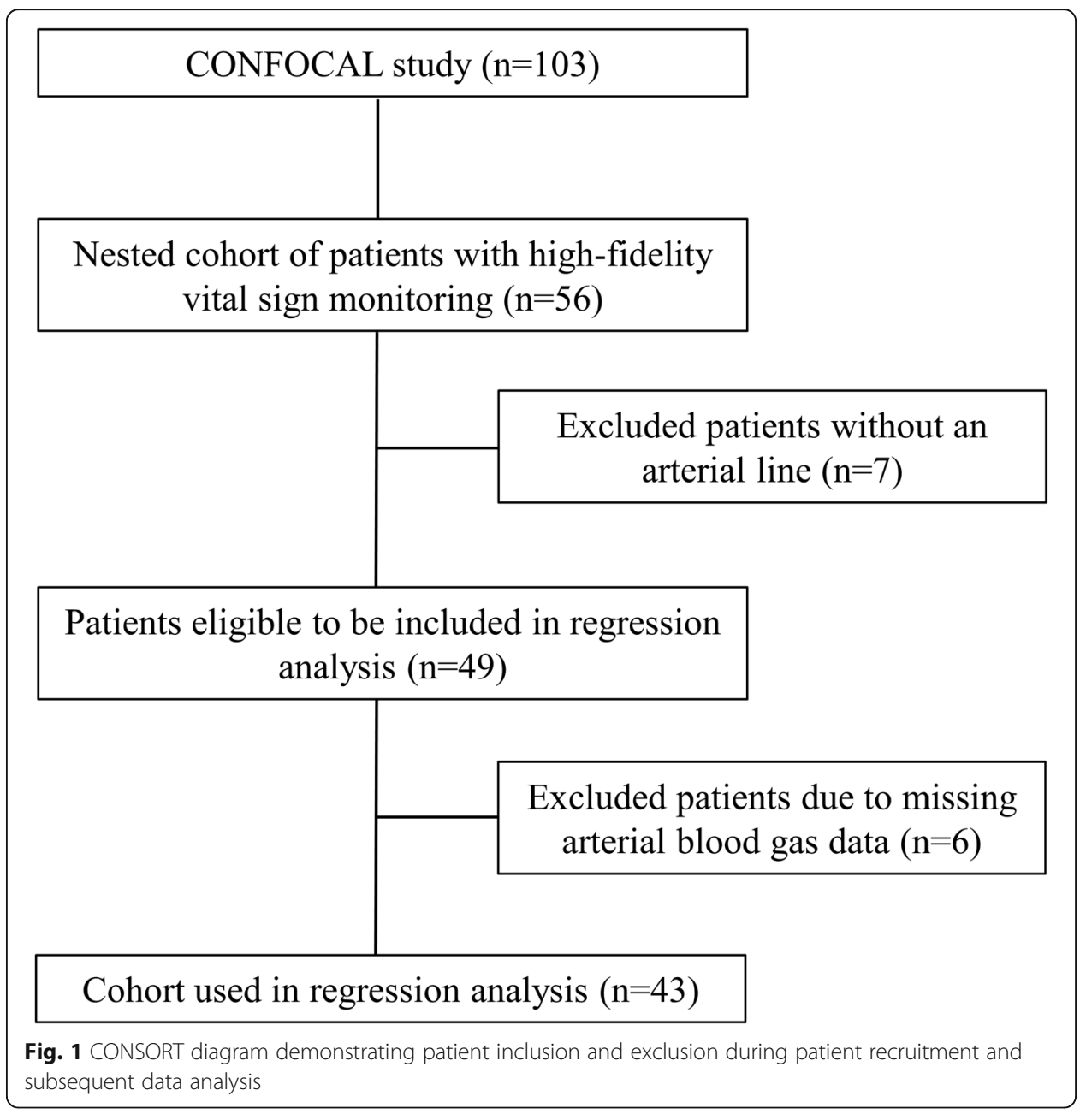


Table 1 Demographics and clinical characteristics

\begin{tabular}{|c|c|}
\hline Characteristic & Nested cohort $(n=43)$ \\
\hline Median age (years, [IQR]) & $68[58.5-79]$ \\
\hline Male gender (no. [\%]) & 29 [67] \\
\hline \multicolumn{2}{|l|}{ Admitting diagnosis (no. [\%]): } \\
\hline Respiratory failure & $13[30]$ \\
\hline Severe sepsis/septic shock & $7[16]$ \\
\hline Cardiac & $7[16]$ \\
\hline Gastrointestinal & $7[16]$ \\
\hline Vascular & $4[9]$ \\
\hline Trauma & $3[7]$ \\
\hline Neurological & 0 \\
\hline Other* & $2[5]$ \\
\hline APACHE score (median, IQR) & $20[16-26]$ \\
\hline \multicolumn{2}{|l|}{ Co-morbidities (no. [\%]): } \\
\hline Cardiac $^{* *}$ & 19 [44] \\
\hline Hypertension & $24[56]$ \\
\hline Respiratory ${ }^{* *}$ & 14 [33] \\
\hline Diabetes & $11[26]$ \\
\hline Active tobacco use & $11[26]$ \\
\hline Heavy alcohol use & $5[12]$ \\
\hline \multicolumn{2}{|l|}{ At time of enrolment (no. [\%]): } \\
\hline Intubated & $41[95]$ \\
\hline Vasoactive agents & $24[56]$ \\
\hline ICU LOS (median [IQR]) & $7[4-13]$ \\
\hline ICU mortality (no. [\%]) & $7[16]$ \\
\hline \multicolumn{2}{|c|}{ Physiological variables (median [IQR]): } \\
\hline MAP $(\mathrm{mmHg})$ & 73.77 [71.04-80.84] \\
\hline HR (bpm) & 85.77 [73.82-99.79] \\
\hline $\mathrm{PaCO}_{2}(\mathrm{mmHg})$ & $40.50[35.20-44.75]$ \\
\hline $\mathrm{PaO}_{2}(\mathrm{mmHg})$ & $86.25[79.75-94.17]$ \\
\hline $\mathrm{pH}(\mathrm{mmHg})$ & $7.37[7.33-7.42]$ \\
\hline $\mathrm{SpO}_{2}(\%)$ & 96.34 [95.23-97.52] \\
\hline $\mathrm{Hb}(\mathrm{g} / \mathrm{L})$ & $102.67[86.00-115.50]$ \\
\hline $\mathrm{rSO}_{2}(\%)$ & $68.41[64.56-72.16]$ \\
\hline
\end{tabular}

MAP mean arterial pressure, $\mathrm{HR}$ heart rate, $\mathrm{PaCO}_{2}$ arterial partial pressure of carbon dioxide, $\mathrm{PaO}_{2}$ arterial partial pressure of oxygen, $\mathrm{SpO}_{2}$ arterial oxygen saturation, $\mathrm{Hb}$ hemoglobin, $\mathrm{rSO}_{2}$ regional cerebral oxygenation

*Other included the following: drug overdose/withdrawal and acute kidney injury

**Cardiac included the following: arrhythmia, prior myocardial infarction, prior cardiac arrest, known coronary artery disease, and/or congestive heart failure

***Respiratory included the following: asthma or COPD

\section{Data collection and analysis}

High-frequency vital sign monitoring was recorded for a median duration of $23.95 \mathrm{~h}$ (IQR, 23.23-24.02), yielding more than 350,000 individual vital sign measurements, including $\mathrm{rSO}_{2}$ recordings. Nearly 20,000 data points were removed due to missing data $(5.5 \%)$, and a further 79 removed due to zero or negative values which were deemed erroneous. We inspected histograms of the distribution of each vital sign to identify 
cutoffs distinguishing true physiologic measures from likely artifacts. Based on these cutoffs, a further 6866 observations (2\%) were removed (see Additional file 4: Figure S4). Compared to the low amount of missing vital sign data, the physiological data obtained from clinically ordered central venous blood gases data were sparse and subsequently excluded from our analysis due to a high degree of missingness. For example, all 43 patients had at least 1 arterial blood gas recording, where 28 patients did not have a venous blood gas sample during the first $24 \mathrm{~h}$ of their ICU stay. Furthermore, the median collected arterial blood gas samples was 2 (IQR, 1-3), whereas the median collected venous blood gas samples was 0 (IQR, 0-1). Median Hb collection throughout the $24 \mathrm{~h}$ of recording was 1 (IQR, 1-2). Therefore, most of the available data was successfully captured for the vital sign monitoring and arterial blood gas data, but a substantial amount of central venous blood gas data was missing or inadequate for subsequent regression analysis.

\section{Physiological determinants of $\mathrm{rSO}_{2}$ during critical illness}

Simultaneous multiple regression analysis

Our simultaneous regression model included all available variables that could influence cerebral oxygen delivery (i.e., age, $\mathrm{HR}, \mathrm{SpO}_{2}, \mathrm{MAP}, \mathrm{pH}, \mathrm{PaO}_{2}, \mathrm{PaCO}_{2}$, and $\mathrm{Hb}$ concentration). This model accounted for a significant proportion of variance in the NIRS-derived $\mathrm{rSO}_{2}$ signal, $R^{2}=0.58, F(8,34)=5.845, p<0.01$. However, $\mathrm{PaCO}_{2}$ and $\mathrm{Hb}$ concentrations were the only significant predictors in the regression model, $b=$ $0.165, t(34)=2.035, p<0.05$, and $b=0.086, t(34)=2.772, p<0.01$, respectively. Both predictors had a positive relationship with $\mathrm{rSO}_{2}$ during the first $24 \mathrm{~h}$ of critical illness. However, several predictors were included in this model, which may have decreased precision of the regression coefficients. This is evidenced by the substantial difference observed between the overall $R^{2}$ and the adjusted $R^{2}$ of 0.48 . The full regression results are shown in Table 2.

\section{Model selection using AICc and multiple regression}

Model selection based on the $\mathrm{AIC}_{\mathrm{c}}$ indicated that the top model (i.e., lowest $\mathrm{AIC}_{\mathrm{c}}$ ) included the following predictors: age, $\mathrm{PaCO}_{2}, \mathrm{Hb}$, and $\mathrm{HR}$. Regression analysis of this selected model accounted for a significant proportion of variance in the $\mathrm{rSO}_{2}$ signal, $R^{2}=0.536, F(4,38)=10.95, p<0.01$, and a comparable adjusted $R^{2}$ of 0.49 was observed for this reduced model. Furthermore, this analysis indicated that the percentage of $\mathrm{rSO}_{2}$ increased significantly with increases in $\mathrm{PaCO}_{2}, b=0.208$, $t(38)=3.062, p<0.01$; $\mathrm{Hb}$ concentration, $b=0.089, t(38)=3.357, p<0.01$; and $\mathrm{HR}$, $b=0.079, t(38)=2.230, p<0.05$ (Table 2 ). In contrast, the percentage of $\mathrm{rSO}_{2}$ significantly decreased as age increased, $b=-0.100, t(38)=-2.329, p<0.05$. The unadjusted effects of the significant predictors on $\mathrm{rSO}_{2}$ can be observed in Fig. 2 . Model comparison using AIC indicated that the selected regression model (i.e., age, $\mathrm{PaCO}_{2}, \mathrm{Hb}$, and $\mathrm{HR}$ ) had a lower AIC when compared to the simultaneous regression model (AIC $=239.64$ and 243.41, respectively), which is further evidenced by the minor difference in variance accounted for by each model, but the selected model includes substantially fewer predictors. 


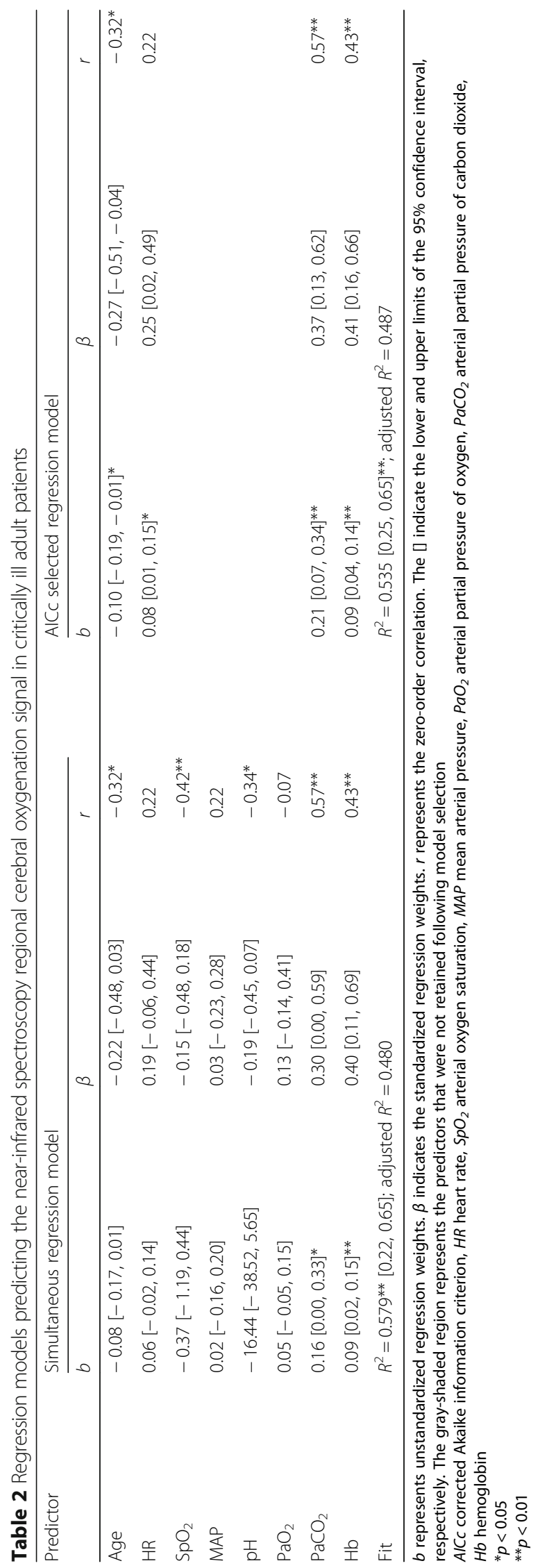




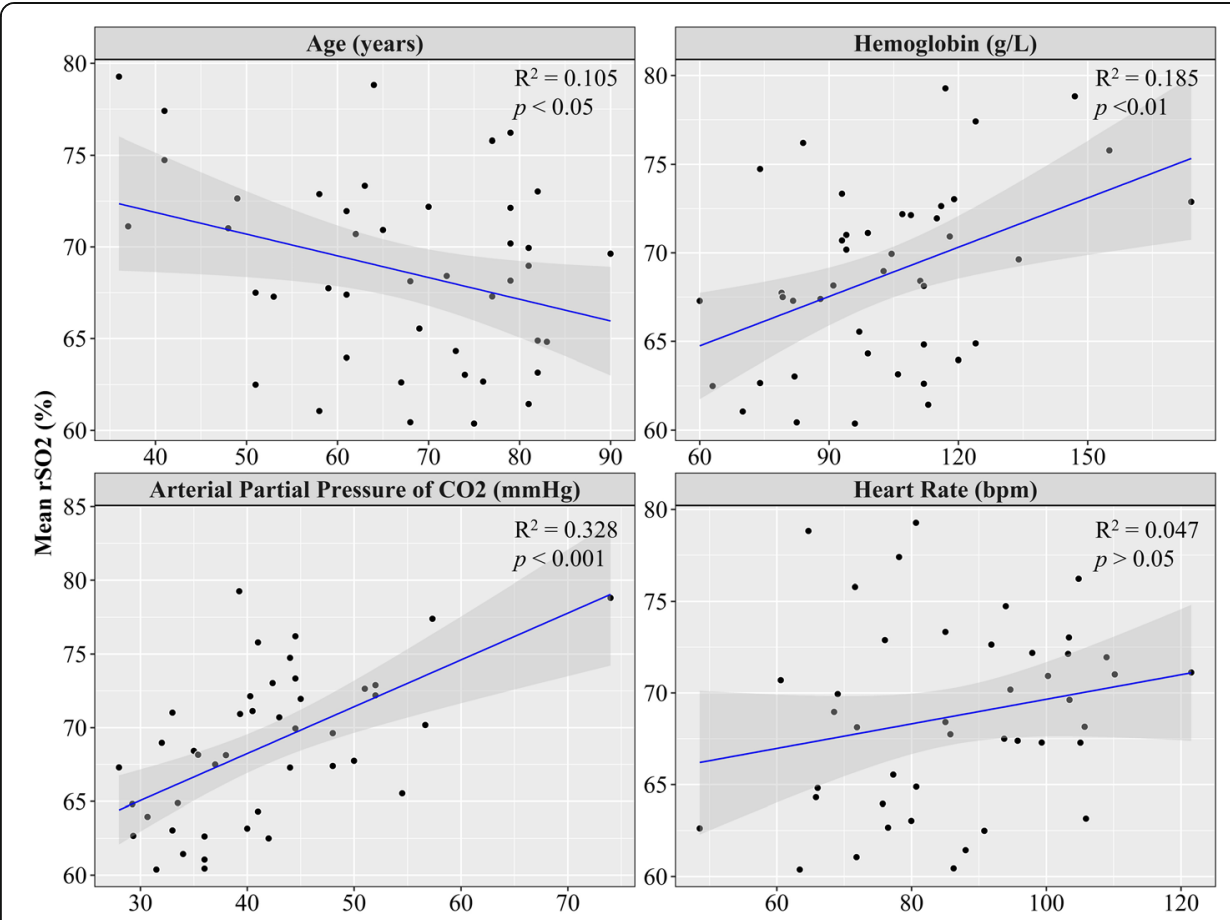

Fig. 2 Scatter plots illustrating the various relationships between regional mean cerebral oxygenation $\left(\mathrm{rSO}_{2}\right)$ recordings and mean levels of various predictors of oxygen delivery (i.e., age, hemoglobin, partial pressure of carbon dioxide, and heart rate). Black data points represent each individual patient with the blue line representing a linear model fit to the data and the gray-shaded region representing the 95\% confidence interval

\section{Discussion}

It has been argued that intraoperative NIRS monitoring should be the standard of care [25], as NIRS has already demonstrated clinically relevant results when used to monitor $\mathrm{rSO}_{2}$ throughout cardiac surgery including fewer incidences of organ dysfunction and shorter ICU stays [26, 27]. However, it is unknown if the determinants of cerebral perfusion during cardiac surgery are similar among critically ill patients who may have dissimilar physiological derangements as part of their illness. We used multivariate modeling to assess the physiological determinants of the NIRS-derived $\mathrm{rSO}_{2}$ signal during the first $24 \mathrm{~h}$ of critical illness in adult patients. Our selected regression model suggested that $\mathrm{PaCO}_{2}, \mathrm{HR}$, and $\mathrm{Hb}$ concentration may be possible therapeutic targets to optimize cerebral oxygenation, as our model accounted for $>50 \%$ of the variance in $\mathrm{rSO}_{2}$. As this analysis was exploratory, we conducted a sensitivity power analysis. Given that we were powered to detect an effect approximately half of what we observed, there is improved confidence that our analysis was not biased for only detecting inflated effect sizes [28]. As this study was based on a single center, our results will need to be further validated in a larger and more diverse cohort of critically ill patients.

\section{Relationship between known determinants of oxygen delivery and $\mathrm{rSO}_{2}$}

As a preliminary step towards demonstrating the utility of NIRS as a surrogate marker of cerebral perfusion in critically ill patients, we assessed the relationship between $\mathrm{rSO}_{2}$ and other variables related to oxygen delivery that are monitored in routine clinical 
practice. It is well accepted that the amount of oxygen delivered to tissues is the product of arterial oxygen content and cardiac output. Arterial oxygen content is determined by $\mathrm{Hb}$ concentration, $\mathrm{SpO}_{2}$, and oxygen dissolved in blood $\left(\mathrm{PaO}_{2} \times 0.003 \mathrm{~mL} /\right.$ $\mathrm{mmHg} \mathrm{O}_{2} / \mathrm{dL}$ blood) [29], whereas cardiac output is determined by HR and stroke volume [30]. Of these determinants of oxygen delivery, we were able to measure $\mathrm{Hb}, \mathrm{HR}$, $\mathrm{SpO}_{2}$, and $\mathrm{PaO}_{2}$.

Red blood cell (RBC) transfusion to improve tissue oxygen delivery is a common ICU intervention administered to approximately $1 / 3$ of critically ill patients [31]. However, this practice still remains controversial due to the potential complications of transfusion (e.g., transfusion-related acute lung injury, altered coagulation, or infections) [32], as well as studies indicating that the restrictive vs liberal use of RBC transfusions may result in similar rates of mortality [33-35] and ischemic events [35]. Importantly, neurological outcomes have not been assessed in these large randomized trials of transfusion thresholds. This issue is further complicated by a lack of understanding of the neurophysiologic effects of RBC transfusion on cerebral oxygenation. In patients with severe traumatic brain injury, mean pre- and post-transfusion $\mathrm{Hb}$ concentrations were significantly different, but this significant difference was not observed for pre- and post- $\mathrm{rSO}_{2}$ recordings [36]. This may have been due to the small sample size and potentially underpowered $(n=19)$ analyses. Our study on critically ill, non-brain-injured patients demonstrated a significant positive association between $\mathrm{Hb}$ concentration and $\mathrm{rSO}_{2}$. This inconsistency between studies may be partially explained by different patient populations being assessed, as patients with traumatic brain injury may have structural etiologies that interfere with the NIRS signal, such as cerebral contusions or hematomas. Our finding raises the possibility that increasing $\mathrm{Hb}$ concentrations within a therapeutic window may be a component of developing a clinical algorithm to optimize $\mathrm{rSO}_{2}$ during critical illness. However, the effects of $\mathrm{RBC}$ transfusions on $\mathrm{rSO}_{2}$, as well as subsequent clinical outcomes, need further analysis.

With regard to cardiac output, we demonstrated a significant positive association between $\mathrm{HR}$ and $\mathrm{rSO}_{2}$. This might suggest that medications that increase heart rate (e.g., dobutamine) could be part of an algorithm to increase cerebral oxygenation. However, it is important to acknowledge that our study did not have data on stroke volume to directly calculate cardiac output. Future studies may wish to utilize non-invasive assessments of cardiac output in order to directly assess the association between cardiac output and $\mathrm{rSO}_{2}$.

Clinicians routinely depend on data derived from pulse oximetry to monitor tissue oxygen saturation in critically ill patients. However, these recordings only provide information regarding the arterial blood content from peripheral tissues. In our study, there was a negative association between $\mathrm{rSO}_{2}$ and $\mathrm{SpO}_{2}$. Furthermore, $\mathrm{SpO}_{2}$ was not identified as a significant predictor of $\mathrm{rSO}_{2}$ when controlling for the other determinants of oxygen delivery. This may suggest that pulse oximetry may inadequately assess cerebral oxygenation, which further argues for the need for routine monitoring of $\mathrm{rSO}_{2}$ at the bedside. Alternatively, this non-significant finding may simply reflect the restricted range of $\mathrm{SpO}_{2}$ levels collected among patients, as arterial oxygen saturation is tightly regulated within the ICU. Due to the small sample size, our analysis may have been underpowered to detect this effect in a multivariate regression model and further analysis is warranted. Furthermore, as mentioned previously, the NIRS signal is mostly 
comprised of venous oxygenation [5]. Therefore, $\mathrm{rSO}_{2}$ may be more related to intracranial venous oxygen saturation, partially explaining the negative association observed with $\mathrm{SpO}_{2}$, and may reflect the interplay between oxygen delivery and consumption. Although $\mathrm{rSO}_{2}$ has been shown to be positively correlated with central venous oxygen saturation previously [37], we had an insufficient sample of central venous oxygen saturation data to assess this relationship in our regression analysis.

In the present study, we did not find a significant association between $\mathrm{rSO}_{2}$ and $\mathrm{PaO}_{2}$. Since the oxygen-hemoglobin dissociation curve becomes relatively flat when oxygen saturation is $>90 \%$ and $\mathrm{PaO}_{2}$ is above $80 \mathrm{mmHg}$ (i.e., sigmoid shape), increases in $\mathrm{PaO}_{2}$ have relatively little impact on saturation/content (i.e., inhaled oxygen will increase $\mathrm{PaO}_{2}$ levels but there will only be a minimal increase in blood oxygen content) [38]. Our cohort had median $\mathrm{SpO}_{2}$ and $\mathrm{PaO}_{2}$ values well above these values mentioned previously, which may partially explain the observed non-significant association. However, had a larger range of values been collected for $\mathrm{PaO}_{2}$ and $\mathrm{SpO}_{2}$, it stands to reason that a significant association(s) may have been observed with $\mathrm{rSO}_{2}$ due to the substantial dependence of oxygen content on $\mathrm{PaO}_{2}$, which would have also been reflected by subsequent changes in $\mathrm{SpO}_{2}$ levels.

\section{$\mathrm{PaCO}_{2}$ may be directly associated with $\mathrm{rSO}_{2}$ in critically ill patients}

Respiratory gases, such as $\mathrm{PaCO}_{2}$, have substantial effects on the radius of cerebral blood vessels (e.g., increases in $\mathrm{PaCO}_{2}$ cause cerebral vasodilation, thus increasing CBF) [39]. However, metabolic acidosis is frequently compensated by spontaneous or controlled hyperventilation during the resuscitation of critically ill patients. As cerebral perfusion is not routinely monitored at the bedside in critically ill patients, the effects of hyperventilation, and subsequent hypocapnia, remain unclear. Since we found a significant positive association between $\mathrm{PaCO}_{2}$ and $\mathrm{rSO}_{2}$, prolonged hyperventilation during critical illness may result in the unintended consequence of compromised cerebral perfusion and potentially secondary neuronal injury.

\section{Physiological parameters NOT associated with $\mathrm{rSO}_{2}: \mathrm{PaO}_{2}$ and MAP}

We describe above the possible explanation(s) for the non-significant association between $\mathrm{rSO}_{2}$ and $\mathrm{PaO}_{2}$. A similar nonsignificant association between MAP and $\mathrm{rSO}_{2}$ was also observed, which may be related to intact cerebral autoregulation as $\mathrm{CBF}$ is preserved through a range of MAP values (i.e., $50-150 \mathrm{mmHg}$ ) [40, 41]. A limitation of the current study is that the integrity of cerebral autoregulation is not captured in this cohort.

\section{Limitations and future directions}

Analyzing the physiological determinants of $\mathrm{rSO}_{2}$ in our cohort of patients was limited by our single-center design and by the small number of patients that underwent high-frequency vital sign recording. Therefore, our findings will need to be validated among a larger cohort of critically ill patients. Additional measures of tissue oxygenation (e.g., $\mathrm{SjvO}_{2}$, lactate) would ideally be incorporated into the regression analysis. However, these lab tests were sent infrequently during the 24-h period of NIRS recording such that the data could not be used. Furthermore, quantifying patient cardiac 
output, rather than recording only heart rate, may provide important information regarding the NIRS association with determinants of oxygen delivery. As stated previously, the NIRS signal is primarily derived from venous circulation (75-80\%) [5]. Our analysis included only arterial blood gas data, which may partially explain why $\sim 50 \%$ of the variance of the NIRS signal was not accounted for by our regression model. Furthermore, given our small sample size, we could not adjust for other potentially relevant covariates that might influence cerebral oxygenation, such as admitting diagnosis, medications, or medical history. Additionally, due to the heterogeneity at which our data was collected (e.g., continuous recording vs clinically ordered), we reduced our data set to a 24-h mean per patient. Future analyses may want to systematically collect all physiological data and conduct a mixed effects regression analysis to account for repeated measurements and potential individual variability among ICU patients. Furthermore, the right and center sensors largely agreed throughout the recording period, which suggests that one sensor in the middle of the forehead may be adequate. However, further research is needed to investigate if the center placement detects focal desaturations that may be clinically meaningful (e.g., right-sided stroke) and better detected using traditional two bilateral sensors, which will be imperative to developing a target algorithm to optimize $\mathrm{rSO}_{2}$. Lastly, age had a significant negative association with $\mathrm{rSO}_{2}$ and will need to be included in future analyses to adjust for this age-related decrease in cerebral perfusion. However, the exact age-related mechanism(s) associated with this decrease in $\mathrm{rSO}_{2}$ is unclear. Despite these limitations, however, we identified three predictors (i.e., $\mathrm{PaCO}_{2}, \mathrm{HR}, \mathrm{Hb}$ ) of the NIRS-derived $\mathrm{rSO}_{2}$ signal that are clinically established determinants of oxygen delivery, which provide evidence that NIRS may be a suitable marker for monitoring cerebral oxygenation during critical illness.

\section{Conclusions}

Our analysis provides evidence that the NIRS-derived $\mathrm{rSO}_{2}$ signal is a viable neuromonitoring modality to assess cerebral oxygenation during critical illness, as this signal was predicted by known and reliable clinical determinants of oxygen delivery during the first $24 \mathrm{~h}$ of critical illness. Further elucidation of the determinants of the $\mathrm{rSO}_{2}$ signal may be useful in developing resuscitation algorithms designed to optimize cerebral oxygenation, an important therapeutic target among critically ill patients. However, clinically relevant covariates will need to be modeled in future analyses.

\section{Endnotes}

${ }^{1}$ Another assumption of regression is linearity for which only one of the predictors violated this assumption, $\mathrm{SpO}_{2}$. Therefore, we also tested a version of the simultaneous model that included the linear and quadratic terms for $\mathrm{SpO}_{2}$. In this model, both linear and quadratic terms for $\mathrm{SpO}_{2}$ were significant predictors of $\mathrm{rSO}_{2}, b=48.47$, $t(33)=2.314, p=0.027, b=-0.26, t(33)=-2.333, p=0.026$, respectively. Because the quadratic term would qualify the linear term, these results indicate that $\mathrm{rSO}_{2}$ is lower at moderate levels of $\mathrm{SpO}_{2}$ than at higher or lower levels of $\mathrm{SpO}_{2}$ arterial. However, complex terms like interactions and polynomials require larger sample sizes for stability. Consequently, we interpret these results with caution and await replication of this finding with a larger sample. 


\section{Additional files}

Additional file 1: Figure S1. Repeated measures Bland Altman plot indicating that the pooled data across the center and right sensors display a high level of agreement. Note. The red dotted lines indicate the $95 \%$ limits of agreement (i.e., the two sensors mean minus $1.96 \mathrm{SD}$ and plus $1.96 \mathrm{SD}$ ). The black line represents the mean (i.e., bias) of recordings across sensors. Black dots represent pooled recordings of regional cerebral oxygenation across 10 intensive care unit patients. As an illustrative example, the Bland Altman analysis indicated that the mean difference (i.e., bias) between the sensors was -0.31 , which indicates that the right sensor on average records $0.31 \%$ higher than the center sensor. Furthermore, the lower and upper limits of agreement $(-7.45 \%$ and $6.84 \%$, respectively) were minor, indicating that the sensors display high agreement. (JPEG $2129 \mathrm{~kb}$ )

Additional file 2: Figure S2. Histograms of high-frequency hemodynamic variable recordings used to remove anomalous data before conducting regression analysis. Diastolic $\mathrm{BP}=$ diastolic blood pressure; MAP = mean arterial pressure; systolic $\mathrm{BP}=$ systolic blood pressure; $\mathrm{rSO}_{2}=$ regional cerebral oxygenation; $\mathrm{SpO}_{2}=$ arterial oxygen saturation. (PDF $37 \mathrm{~kb}$ )

Additional file 3: Figure S3. Matrix of the pooled correlation analysis of all predictors of regional cerebral oxygenation included in the simultaneous regression model. This plot provides visual representation of the associations between various hemodynamic/physiological parameters. The direction of the association is represented by the color (blue= positive; red = negative), and the strength is indicated by shading (dark= strong; light = weak). Each colored square has corresponding text, which represents the $p$ value for the correlation analysis between the column and row parameter. The asterisks indicate significant Pearson correlations coefficients $(p<0.05)$. (JPG $7206 \mathrm{~kb})$

Additional file 4: Figure S4. Bar graph illustrating that amount of missing values per high-frequency vital sign recording. Diastolic $\mathrm{BP}=$ diastolic blood pressure; $\mathrm{MAP}=$ mean arterial pressure; systolic $\mathrm{BP}=$ systolic blood pressure; $\mathrm{rSO}_{2}=$ regional cerebral oxygenation; $\mathrm{SpO}_{2}=$ arterial oxygen saturation. (PDF $22 \mathrm{~kb}$ )

\section{Abbreviations}

95\% Cl: 95\% confidence interval; AlC: Akaike information criterion; AICc: Corrected Akaike information criterion; CBF: Cerebral blood flow; Hb: Hemoglobin concentration; HR: Heart rate; IQR: Interquartile range; MAP: Mean arterial pressure; NIRS: Near-infrared spectroscopy; $\mathrm{PaCO}_{2}$ : Arterial partial pressure of carbon dioxide; $\mathrm{PaO}_{2}$ : Arterial partial pressure of oxygen; $\mathrm{RBC}$ : Red blood cell; $\mathrm{rSO}_{2}$ : Regional cerebral oxygenation; $\mathrm{SjvO}_{2}$ : Jugular venous oxygen saturation; $\mathrm{SpO}_{2}$ : Peripheral oxygen saturation

\section{Acknowledgements}

The authors would like to thank the study coordinators, Ms. Miranda Hunt, Ms. Nicole O'Callahan, Ms. llinca Georgescu, and Mrs. Tracy Boyd. We would also like to thank the entire ICU staff at Kingston Health Sciences Centre.

\section{Funding}

This work was funded by the Physician Services Incorporated and the Southeastern Ontario Academic Medical Organization New Clinician Scientist Program for which JGB was the recipient. The funding agencies had no role in the design of this study, data collection, or data analysis.

\section{Availability of data and materials}

The datasets used and/or analyzed during the current study are available from the corresponding author on reasonable request.

\section{Authors' contributions}

MDW participated in study design, statistical plan, data collection, data analysis, and drafting of the manuscript. JAJ participated in the statistical plan, data analysis, reproducing study results and sensitivity power analysis, and drafting of the manuscript. DMM participated in study design, data analysis, and drafting of the manuscript. JGM participated in study design and drafting of the manuscript. JGB is the primary investigator. He participated in study design and drafting of the manuscript. All authors read and approved the final manuscript.

\section{Ethics approval and consent to participate}

The Queen's University and Affiliated Hospitals Health Sciences Research Ethics Board approved this study protocol, which included deferred consent for $24 \mathrm{~h}$ due to the non-invasive nature of the monitoring device. Informed consent was obtained from all subjects or their proxy before enrollment.

\section{Consent for publication}

Not applicable.

\section{Competing interests}

JGM is the scientific director of the Canadian Frailty Network. JGB receives a stipend from the Trillium Gift of Life Network to support his role as the Hospital Donation Support Physician. All other authors declare that they have no competing interests.

\section{Publisher's Note}

Springer Nature remains neutral with regard to jurisdictional claims in published maps and institutional affiliations. 


\section{Author details}

'Centre for Neuroscience Studies, Queen's University, 18 Stuart St, Botterell Hall, Kingston, ON, Canada. ${ }^{2}$ Department of Psychology, Queen's University, 62 Arch Street, 318 Craine Hall, Kingston, ON, Canada. ${ }^{3}$ Department of Critical Care Medicine, Queen's University, Rm 22.2.359 Davies 2, Kingston General Hospital, 76 Stuart St, Kingston, ON K7L 2V7, Canada. ${ }^{4}$ Department of Medicine, Queen's University, Rm 4.5.310 Watkins C, Kingston General Hospital, 76 Stuart St, Kingston, ON, Canada.

\section{Received: 4 February 2019 Accepted: 7 April 2019}

\section{Published online: 02 May 2019}

\section{References}

1. Dellinger RP, Levy MM, Rhodes A et al (2013) Surviving Sepsis Campaign: international guidelines for management of severe sepsis and septic shock. Intensive Care Med 39:165-228. https://doi.org/10.1007/s00134-012-2769-8.

2. Sharshar T, Annane D, de la Grandmaison GL et al (2004) The neuropathology of septic shock. Brain Pathol 14:21-33.

3. Sharshar T, Citerio G, Andrews PJD et al (2014) Neurological examination of critically ill patients: a pragmatic approach. Report of an ESICM expert panel. Intensive Care Med 40:484-495. https://doi.org/10.1007/s00134-014-3214-y.

4. Scott JP, Hoffman GM (2014) Near-infrared spectroscopy: exposing the dark (venous) side of the circulation. Paediatr Anaesth 24:74-88. https://doi.org/10.1111/pan.12301.

5. Marin T, Moore J (2011) Understanding near-infrared spectroscopy. Adv Neonatal Care 11:382-388. https://doi.org/10. 1097/ANC.0b013e3182337ebb.

6. Kim MB, Ward DS, Cartwright CR et al (2000) Estimation of jugular venous O2 saturation from cerebral oximetry or arterial O2 saturation during isocapnic hypoxia. J Clin Monit Comput 16:191-199.

7. McLeod AD, Igielman F, Elwell C et al (2003) Measuring cerebral oxygenation during normobaric hyperoxia: a comparison of tissue microprobes, near-infrared spectroscopy, and jugular venous oximetry in head injury. Anesth Analg 97:851-856.

8. Taussky P, O'Neal B, Daugherty WP et al (2012) Validation of frontal near-infrared spectroscopy as noninvasive bedside monitoring for regional cerebral blood flow in brain-injured patients. Neurosurg Focus 32:E2. https://doi.org/10.3171/ 2011.12.FOCUS11280

9. Wood MD, Song A, Maslove D, Ferri C, Howes D, Muscedere J, Boyd J Brain tissue oxygenation in patients with septic shock: a feasibility study. Can J Neurol Sci. 2016;43(1):65-73. https://doi.org/10.1017/cjn.2015.280. Epub 2015 Sep 4. PMID: 26338150.

10. Denault A, Deschamps A, Murkin JM (2007) A proposed algorithm for the intraoperative use of cerebral near-infrared spectroscopy. Semin Cardiothorac Vasc Anesth 11:274-281. https://doi.org/10.1177/1089253207311685.

11. Deschamps A, Lambert J, Couture P et al (2013) Reversal of decreases in cerebral saturation in high-risk cardiac surgery. J Cardiothorac Vasc Anesth 27:1260-1266. https://doi.org/10.1053/j.jvca.2013.01.019.

12. Deschamps A, Hall R, Grocott $\mathrm{H}$ et al (2016) Cerebral oximetry monitoring to maintain normal cerebral oxygen saturation during high-risk cardiac surgery: a randomized controlled feasibility trial. Anesthesiology 124:826-836. https:// doi.org/10.1097/ALN.0000000000001029.

13. Wood MD, Maslove DM, Muscedere JG et al (2017) Low brain tissue oxygenation contributes to the development of delirium in critically ill patients: a prospective observational study. J Crit Care 41:289-295. https://doi.org/10.1016/j.jcrc. 2017.06.009.

14. Wood MD, Maslove D, Muscedere J et al (2016) Assessing the relationship between brain tissue oxygenation and neurological dysfunction in critically ill patients: study protocol. Int J Clin Trials 3:98-105.

15. Gregory AJ, Hatem MA, Yee K, Grocott HP (2016) Optimal placement of cerebral oximeter monitors to avoid the frontal sinus as determined by computed tomography. J Cardiothorac Vasc Anesth 30:127-133. https://doi.org/10.1053/j.jvca. 2015.07.004.

16. Taillefer M-C, Denault AY (2005) Cerebral near-infrared spectroscopy in adult heart surgery: systematic review of its clinical efficacy. Can J Anaesth 52:79-87. https://doi.org/10.1007/BF03018586.

17. Chen L, Dubrawski A, Wang D et al (2016) Using supervised machine learning to classify real alerts and artifact in online multisignal vital sign monitoring data. Crit Care Med 44:e456-e463. https://doi.org/10.1097/CCM.0000000000001660.

18. R Core Team (2017) R: A Language and Environment for Statistical Computing.

19. Buijs PC, Krabbe-Hartkamp MJ, Bakker CJ et al (1998) Effect of age on cerebral blood flow: measurement with ungated two-dimensional phase-contrast MR angiography in 250 adults. Radiology 209:667-674. https://doi.org/10.1148/ radiology.209.3.9844657.

20. Barton K (2017) MuMIn: multi-model inference.

21. Hocking RR (1976) A biometrics invited paper. The analysis and selection of variables in linear regression. Biometrics 32 : 1. https://doi.org/10.2307/2529336

22. Hurvich CM, Tsai C-L (1989) Regression and time series model selection in small samples. Biometrika 76:297. https://doi. org/10.2307/2336663.

23. Akaike H (1974) A new look at the statistical model identification. IEEE Trans Automat Contr 19:716-723. https://doi.org/ 10.1109/TAC.1974.1100705.

24. Faul F, Erdfelder E, Lang A-G, Buchner A (2007) G*Power 3: a flexible statistical power analysis program for the social, behavioral, and biomedical sciences. Behav Res Methods 39:175-191. https://doi.org/10.3758/BF03193146.

25. Bevan PJW (2015) Should cerebral near-infrared spectroscopy be standard of care in adult cardiac surgery? Hear Lung Circ 24:544-550. https://doi.org/10.1016/j.hlc.2015.01.011.

26. Murkin JM, Adams SJ, Novick RJ et al (2007) Monitoring brain oxygen saturation during coronary bypass surgery: a randomized, prospective study. Anesth Analg 104:51-58. https://doi.org/10.1213/01.ane.0000246814.29362.f4.

27. Murkin JM, Arango M (2009) Near-infrared spectroscopy as an index of brain and tissue oxygenation. Br J Anaesth 103 Suppl:i3-13. doi: https://doi.org/10.1093/bja/aep299.

28. Button KS, loannidis JPA, Mokrysz C et al (2013) Power failure: why small sample size undermines the reliability of neuroscience. Nat Rev Neurosci 14:365-376. https://doi.org/10.1038/nrn3475. 
29. Maloney-Wilensky E, Le Roux P (2010) The physiology behind direct brain oxygen monitors and practical aspects of their use. Childs Nerv Syst 26:419-430. https://doi.org/10.1007/s00381-009-1037-x.

30. King J, Lowery DR. Physiology, Cardiac Output. [Updated 2019 Jan 3]. In: StatPearls [Internet]. Treasure Island (FL): StatPearls Publishing; 2019. Available from: https://www.ncbi.n/m.nih.gov/books/NBK470455/.

31. Lelubre C, Vincent J-L (2011) Red blood cell transfusion in the critically ill patient. Ann Intensive Care 1:43. https://doi. org/10.1186/2110-5820-1-43.

32. Maxwell MJ, Wilson MJA (2006) Complications of blood transfusion. Contin Educ Anaesth Crit Care Pain 6:225-229. https://doi.org/10.1093/bjaceaccp/mkl053.

33. Hébert PC, Wells G, Blajchman MA et al (1999) A multicenter, randomized, controlled clinical trial of transfusion requirements in critical care. N Engl J Med 340:409-417. https://doi.org/10.1056/NEJM199902113400601.

34. Mazer CD, Whitlock RP, Fergusson DA et al (2017) Restrictive or liberal red-cell transfusion for cardiac surgery. N Engl J Med 377:2133-2144. https://doi.org/10.1056/NEJMoa1711818.

35. Holst LB, Haase N, Wetterslev J et al (2014) Lower versus higher hemoglobin threshold for transfusion in septic shock. N Engl J Med 371:1381-1391. https://doi.org/10.1056/NEJMoa1406617.

36. McCredie VA, Piva S, Santos M et al (2017) The impact of red blood cell transfusion on cerebral tissue oxygen saturation in severe traumatic brain injury. Neurocrit Care 26:247-255. https://doi.org/10.1007/s12028-016-0310-6.

37. Al Tayar A, Abouelela A, Mohiuddeen K (2017) Can the cerebral regional oxygen saturation be a perfusion parameter in shock? J Crit Care 38:164-167. https://doi.org/10.1016/j.jcrc.2016.11.006

38. Collins J-A, Rudenski A, Gibson J et al (2015) Relating oxygen partial pressure, saturation and content: the haemoglobinoxygen dissociation curve. Breathe (Sheffield, England) 11:194-201. https://doi.org/10.1183/20734735.001415.

39. Lipp LL (2014) Brain perfusion and oxygenation. Crit Care Nurs Clin North Am 26:389-398. https://doi.org/10.1016/j.ccell. 2014.04.008.

40. Nemani VM, Manley GT (2004) Brain tissue oxygen monitoring: physiologic principles and clinical application. Oper Tech Neurosurg 7:2-9. https://doi.org/10.1053/j.otns.2004.04.002.

41. Dunn IF, Ellegala DB, Fox JF et al (2006) Principles of cerebral oxygenation and blood flow in the neurological critical care unit. Neurocrit Care 4:77-82.

\section{Submit your manuscript to a SpringerOpen ${ }^{\circ}$ journal and benefit from:}

- Convenient online submission

- Rigorous peer review

- Open access: articles freely available online

- High visibility within the field

- Retaining the copyright to your article

Submit your next manuscript at $\gg$ springeropen.com 